\title{
Focused low-energy extracorporeal shock waves with distally symmetric polyneuropathy (DSPNP): A pilot study
}

\author{
H. Lohse-Busch ${ }^{\mathrm{a}, *}$, E. Marlinghaus ${ }^{\mathrm{b}}$, U. Reime ${ }^{\mathrm{a}}$ and U. Möwis ${ }^{\mathrm{a}}$ \\ ${ }^{a}$ Outpatient Department for Manual Medicine - Movement Disorder Center Rheintalklinik, Bad Krozingen, Germany \\ ${ }^{\mathrm{b}}$ Storz Medical AG, Tägerwilen, Switzerland
}

\begin{abstract}
.
BACKGROUND: Incidental observations led to the question whether ESWT could alleviate the still difficult-to-improve symptoms of DSPNP.

METHODS: In a pilot study, out of an original 24 patients with DSPNP, 10 patients with diabetes mellitus were excluded because of their inhomogeneous performances. Of the 14 patients remaining, 6 received one sham treatment at the beginning. All 14 patients were then treated with ESWT to the soles of the feet using the Duolith ${ }^{\circledR}$ shock wave generator (Storz Medical) 3 times weekly for 2 weeks. The assessments were carried out before and after the sham treatment, the first ESWT (question: before and after the first ESWT or only after?) and after 2, 4 and 8 weeks.

RESULTS: The placebo treatment did not influence pain or paraesthesia. After the 2 weeks of ESWT, intensity decreased from $100 \%$ to $23.6 \%$, rising again after 8 weeks to $45.7 \%$ of the original state $(p<0.01)$. The placebo treatment did however have a great effect on walking abilities. The results of ESWT did not become significant until the 8th week. Step length improved by $14.6 \%(p<0.001)$, walking speed by $24.8 \%(p<0.001)$ and time of dual support during the stance phase of the gait declined by $12.2 \%(p<0.009)$.
\end{abstract}

CONCLUSIONS: Despite the small number of cases, it appears that ESWT can alleviate some of the symptoms of DSPNP.

Keywords: Extracorporeal shock wave therapy, polyneuropathy, pain, walking kinematics, paraesthesia

\section{Background}

Patients with distally symmetric polyneuropathy (DSPNP) suffer from individually different symptoms. In the majority of cases, only the lower extremities are affected. Symptoms include paraesthesia, mainly formication, a feeling of walking on cotton wool, furriness and numbness of the feet, pain of various qualities, including burning feet, and limitation or loss of tem-

\footnotetext{
*Address for correspondence: H. Lohse-Busch, Outpatient Department for Manual Medicine - Movement Disorder Center Rheintalklinik, Im Rheintal 5, D-79189 Bad Krozingen, Germany. Tel.: +49 7633408 836; Fax: +49 7633408 842; E-mail: lohse-busch@amm-rheintalklinik.de.
}

perature discrimination. At least in the early stages, these symptoms can be relieved for limited periods by walking.

The disturbances of sensation are accompanied by the motor function disturbances that are sometimes closely associated with them: muscular atrophy, reduced muscle strength and a tendency to experience night-time pain in the feet, as well as cramps in the muscles of the calves and in the bending muscles of the toes. In addition to muscular weakness, a general disruption of spatial awareness (proprioception) with characteristically wooden movements, a shortening of steps, lack of assurance while walking and a tendency to stumble are also experienced. 
Loss of hair on the extensor sides of the lower leg, diminishment to the point of absence of the formation of calluses on the feet and generally thinned-out, vulnerable skin are all indicative of deficits in the vegetative nervous system. When experienced in conjunction with microangiopathy, a disturbed sensibility leads to torpid ulcers that are not infrequently to be found at the beginning of a process, culminating in amputations in the area of the lower extremities.

The main cause of DSPNP is the metabolic syndrome of Type II diabetes and alcohol abuse. However, toxic medications (e.g. in chemotherapy), commercial and environmental toxins also come into question as possible sources of the disease. The cause of the disease is often not able to be determined.

The disease begins slowly as a rule and then develops progressively. It can also inexplicably come to a stop or even go into remission in isolated cases.

The symptoms can be improved only to a certain extent through normalisation of the metabolic syndrome, abstinence from alcohol and avoidance of other toxic substances. Furthermore, no effective treatment method exists that has a causal effect on the disease. Experiments with medications are usually unsuccessful. The only exception is B12 substitution for pernicious anaemia. Physiotherapy results in a very limited improvement of symptoms. Movements of the affected limbs have a short-term palliative effect. Patients should, on the other hand and to the greatest extent possible, maintain their proprioception through continuous training in order to take advantage of the effects of this on the sensory and motor systems in order to counteract a tendency to fall.

ESWT has been used for the last 20 years for orthopaedic problems such as plantar fasciitis (Speed 2003), tennis elbow (Melikyan, 2003), calcific tendinosis of the shoulder (Mouzopoulos, 2007) and chronic achilles tendinopathy (Al-Abbad, 2013). The strong anti-inflammatory and therefore analgesic action is very well known (Mariotto, 2009).

Low-energy focused extracorporeal shock waves (ESW) have been offering new possibilities for neurological rehabilitation for more than 10 years. The results of the treatment of patients with spastic movement disabilities (Amelio, 2010; Lohse-Busch, 1997; Manganotti, 2005; Troncatia, 2013) encourage the idea that this form of treatment not only has an effect on musculature but also promotes proprioception. The results of ESWT on nerve regeneration in the rat (Hausner, 2012; Mense, 2013) are encouraging with respect to the idea of possible nerve repair.
The working theory proposed in this connection is that extracorporeal shock waves might be able to alleviate pain and paraesthesia on the one hand, and walking speed, step length and dual support of the legs during the stance phases on the other. It was therefore a logical step to carry out a treatment experiment among patients with DSPNP.

\section{Method and patients}

\subsection{Ethics commission}

The Ethics Commission of Freiburg University Medical Center informed us that they had no reservations against the performance of these therapy sessions.

\subsection{Inclusion criteria}

Consistent with the character of a pilot study, no distinction was made between the various causes, degrees of severity and stages of the disease. The only decisive factors were the establishment of diagnosis through at least four objective signs and four subjective symptoms and the severity of the patient's suffering that led him or her to participate in the investigation (see Table 1).

In response to an announcement posted in the waiting area of the clinic, 24 patients with DSPM volunteered to participate over an 8-week period, including 10 patients with diabetes mellitus of various degrees of severity. The original intention was to investigate DSPNP in all of its forms under the auspices of a pilot study.

It became evident at the time the results were evaluated, however, that the patients with diabetes mellitus made it impossible to arrive at statistically unambiguous results. Patients who did not suffer from this disease could, however, be included very readily in the statistical calculations. We therefore subsequently decided to exclude patients with diabetes mellitus from this study in order to have them undergo later investigations with larger numbers of patients.

In the end, 14 patients remained who met the criteria for inclusion. The nine men and five women, with an average age of 67.7 (44-84) years, suffered from various forms and degrees of severity of DSPNP (Table 2).

\subsection{Exclusion criteria}

Necessity of aids for walking (walking sticks, leg braces, etc.), painful arthritic processes of the lower extremities as an additional hindrance to walking, and subsequently patients with diabetes mellitus. 


\subsection{Procedure}

All patients declared their written consent to participate in the study after receiving written and oral information about it.

A clinical baseline examination was performed at the start to select the patients who met the inclusion criteria. Step length, speed of walking and times of the load on the lower limbs during the stance phase were measured with the aid of a mat equipped with electronic sensors (GaitRite mat ${ }^{\circledR}$ ). This task was performed by an assistant who has had years of training in the use of this device. The ESWT were performed by one physician, while the supervision of the blinding and the data transfer was undertaken by a different physician who had no further involvement with the treatments otherwise. The statistical evaluation was performed out-of-house.

Storz Medical's Duolith ${ }^{\circledR}$ was used to administer the ESWT. The sham treatments were carried out with the help of a CD player that was hidden underneath the device. The device emitted only the characteristic cracking noises, but not the shock waves themselves.

The paraesthesia and pains of the patients varied widely, ranging from feelings of numbness to sensations of pins-and-needles to tearing pain. The patients were therefore asked to use the number 100 to describe their condition before the treatments.

In order to obtain an overview of a possible placebo effect, the first six patients were subjected to one initial sham treatment. Out of consideration for the reasonableness of the demands made on the patients, only one single blinded sham treatment was carried out for the patient and evaluators. The sham treatment and the first active treatment were administered the same day.

The treatments were administered immediately after the measurements of kinematics made with the GaitRite mat and the assessment of pain, paraesthesia and feelings of discomfort with the visual analogue scale, and it was followed in turn by another measurement. These assessments were also carried out after 2 weeks (i.e. at the end of the treatment series), after 4 and after 8 weeks.

Walking speed, step length and time of dual support in the stance phase were evaluated as the most important kinematic parameters for the patient. The shortening of the time of dual support during the stance phase corresponds to the relative lengthening of the swing phase while walking. A short time interval of dual support is a measure of increasing equilibrium and assurance while walking.

The patients received one treatment with focused ESW each on Monday, Wednesday and Friday over the course of the two weeks. 2000 shock waves were applied at each session to the soles of the feet with energy flux densities ranging from $0.08 \mathrm{~mJ} / \mathrm{mm}^{2}$ to 0.15 $\mathrm{mJ} / \mathrm{mm}^{2}$, depending on individual tolerance. The focus was set in such a way that, in addition to the receptors of the skin, the densely innervated structures of the musculi interossei and lumbricales, the musculus quadratus plantae and the sustentaculum tali were also accessed. The therapy source was moved constantly back and forth across the tissue to be treated.

The signed rank test was used for the statistical evaluation of the development of feelings of discomfort caused by DSPNP because no normal distribution resulted from the measured values. All other statistical calculations were undertaken in accordance with the paired $t$-test in direct comparison with the measurements made before and after the events to be compared.

\section{Results}

The placebo treatment had no influence on pain, paraesthesia or feelings of discomfort. During the twoweek treatment series with ESWT, however, these complaints decreased in their intensity to as low as $23.6 \%$, when measured against their original levels. Afterwards, during the observation interval following the treatments, there was a gradual resurgence of the feelings of discomfort over the course of six weeks, to an intensity of $45.7 \%$ of the original state (Table 3, Fig. 1).

Considerable difficulties arose in connection with the statistical evaluation of the parameters. Not all of the measurements carried out during the intervals yielded statistically unambiguous results. The significance of the statements did not become evident until the end of the eight-week observation period.

Even though the placebo treatment had no appreciable effect on pain and paraesthesia, it did, however, have a significant effect on the kinematic parameters. Step length increased by $11.1 \%$ and speed by $18.9 \%$ as a result of the one-time placebo treatment. The time of dual support during the stance phase of the gait decreased by $6.1 \%$.

The group that was originally treated only once with the placebo $(n=6)$ was subsequently treated with ESWT, the same as with all of the other patients. This resulted in a group of 14 patients with active treatment.

If, however, one tracks the placebo group separately over the entire eight weeks, the result improves in comparison with the one-time placebo treatment through the subsequent active treatment by $19.9 \%(p=0.06)$ for step 
100

Reduction of paresthesia and pain

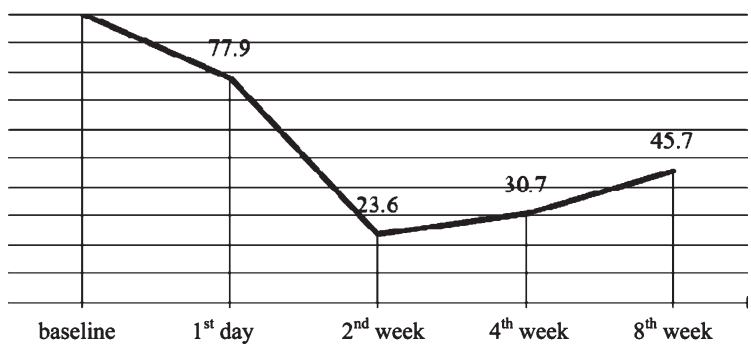

Fig. 1. Reduction of paraesthesia, feelings of discomfort and pain in the area of the lower extremities during ESWT and the slow resurgence of these symptoms during the subsequent six-week observation period.

length, $37.1 \%(p=0.032)$ for walking speed and $12.6 \%$ $(p=0.463)$ for the reduction of the duration of dual support. With this level of significance the probability of error is high.

The group as a whole (i.e. including the placebo group as well), showed statistically clearer results with the improvement of step length during the eight-week observation period by $14.6 \%(p<0.001)$ Fig. 2 , and the increase in walking speed of $24.8 \%(p<0.001)$ Fig. 3. The time of dual support declined by $12.2 \%(p=0.09)$ Fig. 4. This is indicative of only a tendency.

Following the two-week treatment with ESWT, starting from a maximum improvement of $23.6 \%$ for feelings of discomfort and pain, a slight increase with respect to the patients' complaints was experienced over the course of the six-week observation period, namely to $45.7 \%$ of the original state. Step length, walking speed and the duration of the dual-support time also improved during the two-week treatment, but then worsened during the following two weeks, only to improve once again at the end of the overall observation period of eight weeks. Figures 2-4 show the same pattern for all three parameters.

\section{Discussion}

Treatments involving large numbers of high-energy focused ESW applied at different points destroy nerves (Kato, 2007; Wu, 2008). Depending on the energy applied, however, these also lead to the regeneration of nerves in animal models (Mense, 2013; Wu, 2008). It has previously been discovered that low-energy ESW stimulate not only the production of endothelial nitric oxides (eNOs) (Mariotto, 2005) but also angiogenesis (Gutersohn, 2000; Ito, 2009; Ma, 2005) and neuroge-

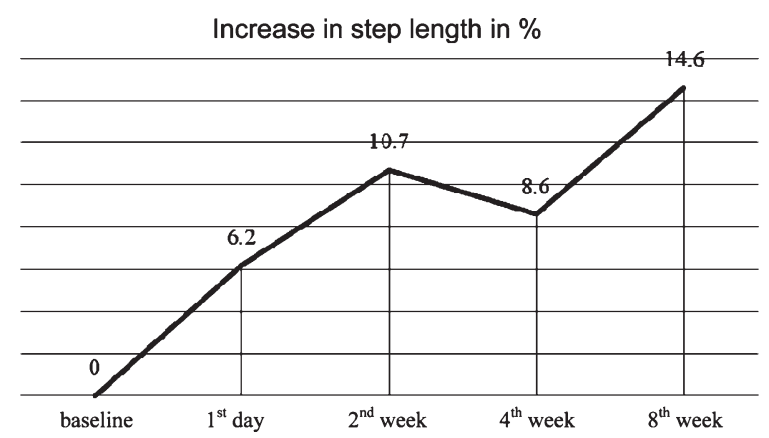

Fig. 2. Increase in step length, left, during ESWT with further improvements at the end of the observation period.

Increase in walking speed in \%

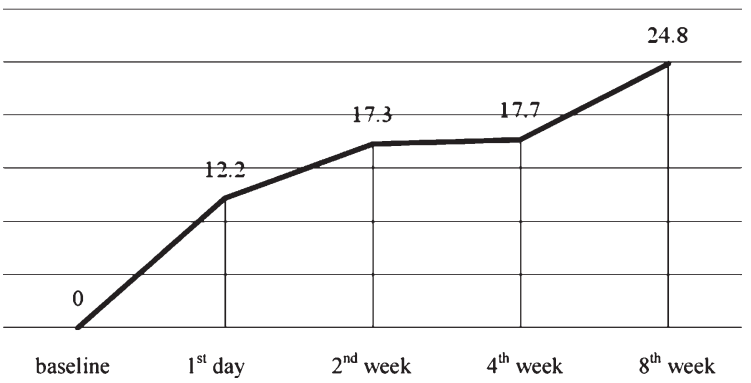

Fig. 3. Following the initial improvement of walking speed during ESWT, there may possibly be later training effects at work.

Reduction of the time of dual support in \%

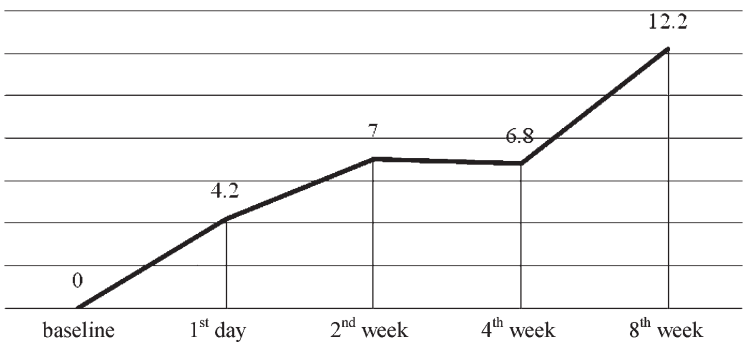

Fig. 4. The period of dual support during the stance phase of the gait became shorter as an indirect measurement of assurance while walking.

nesis (Jin, 2006; Sun, 2006; Widenfalk, 2003) through the vascular endothelial growth factor (VEGF). Neurogenesis is also promoted by the FGF-2 (fibroblast growth factor) (Laird, 1995; Mudò, 2009), which is also activated by ESW. The stimulation of the endothelial lymphatic growth factor (ELGF) has a positive effect on lymph angiogenesis (Kubo, 2010; Serizawa, 2011). Following application of ESW to a necrosis of the hip, a systemic increase in the concentration of NO, VEGF 
Table 1

Quantifiable and subjective clinical inclusion criteria

Objective signs
Sensibility impairments
Limitation of pallaesthesia
Limitation of sense of touch
Reduction/loss of sense of heat/cold
Limitation of graphaesthesia
Limitation of pain sensation (pinwheel)
Electrophysiological signs (nerve conduction velocity, neurography)
Trophic disorders
Loss of hair on the lower leg over a dermatome
Loss of callusing on the sole of the foot
Paper-thin, dry skin on the lower leg
Absence of sweat on the feet
Myatrophy of the musculi interossei, the peroneal group
Trophic ulcers
Pain and paraesthesia
Subjective symptoms

Feeling of numbness

Reduction/loss of sense of heat/cold "Ice-cold" feet and/or burning feet at objectively normal temperatures

Night-time itching of the lower leg

Subjective muscle weakness of the lower legs

Disruption of proprioception

Numbness

Pins-and-needles paraesthesia in the feet

Abrupt shooting pain in the lower leg/feet for seconds/minutes

Night-time muscle cramps

Other unexplainable muscle and foot pain

Limited proprioception and equilibrium (tightrope walk, one-leg-stand) Uncertainty in darkness, tendency to stumble

Table 2

Aetiologies of DSPNP in the patients investigated

\begin{tabular}{lc}
\hline Aetiology & $n$ \\
\hline Paraneoblastic & 2 \\
Toxic (PCB, Interferon) & 2 \\
Monoclonal gammopathy & 1 \\
Malabsorption with Crohn's disease & 1 \\
Uncertain cause & 8 \\
& 14 \\
\hline
\end{tabular}

and FGF in serum was documented over a 4 to 8 week period, and it was not until three months later that it was no longer significantly elevated (Wang, 2011). In the animal model, VEGF promotes functional regeneration and inhibits secondary degeneration following experimental contusions of the spinal cord (Widenfalk, 2003).

Previous experience with the alleviation of the complaints associated with DSPNP using ESWT ultimately led to this investigation. Nevertheless, unexpected results emerged. On the one hand, the measurement values recorded with the diabetic patients could not be lined up statistically due to the breadth of their spread. During the preparation time for this study, however, it was precisely the results that had been achieved among diabetics that resulted in the original decision to carry out this investigation in the first place.

In the meantime, it appears to be the case that the number of shock waves that are applied to the soles of
Table 3

Development of pain, paraesthesia and feelings of discomfort immediately after placebo treatment and during follow-up observation

\begin{tabular}{|c|c|c|c|c|c|c|}
\hline \multicolumn{3}{|c|}{ Feeling of discomfort } & \multirow{2}{*}{$\begin{array}{c}\text { after } \\
\text { 1st ESWT }\end{array}$} & \multicolumn{3}{|c|}{ Follow-up observation } \\
\hline & Baseline & $\begin{array}{c}\text { After } \\
\text { placebo }\end{array}$ & & $\begin{array}{l}2 \text { nd } \\
\text { week }\end{array}$ & $\begin{array}{c}\text { 4th } \\
\text { week }\end{array}$ & $\begin{array}{c}\text { 8th } \\
\text { week }\end{array}$ \\
\hline $\begin{array}{l}n=6 \\
n=14\end{array}$ & 100 & $95 p<0.5$ & 77.9 & 23.6 & 30.7 & $\begin{array}{c}45.7 \\
p<0.001\end{array}$ \\
\hline
\end{tabular}

the feet in order to achieve the desired effects must be considerably higher for diabetics than for other patients. A correspondingly extended investigation of DSPNP among diabetics would be desirable.

The second surprise was the high placebo effect on the motor parameters, although no similar effect on the feelings of discomfort and pain of DSPNP was experienced. That may be due to the fact that, whereas one can walk faster when one makes more of an effort while under the influence of a placebo, one cannot suppress pain by this act of will.

The alleviation of pain was experienced immediately after the active treatment. A continuous reduction in the complaints occurred during treatment, down to approximately one quarter of the original intensity. The feelings of discomfort undergo a slight increase following the end of treatment with ESW. After the eighth week, 
Table 4

Development of step length, walking speed and dual support of the legs in the stance phase during treatment and at the end of the eight-week observation period. The initial placebo group was merged into the group with active treatment after the sham treatment

\begin{tabular}{|c|c|c|c|c|c|c|}
\hline & \multicolumn{4}{|c|}{ Treatment interval } & \multicolumn{2}{|c|}{ Follow-up observation } \\
\hline & & After placebo & 1st day & 2nd week & 4th week & 8th week \\
\hline \multirow[t]{2}{*}{ Step length left } & placebo group $n=6$ & $11.1 p=0.041$ & & & & $19.9 p=0.061$ \\
\hline & active treatment $n=14$ & & 6.2 & 10.7 & 8.6 & $14.6 p<0.001$ \\
\hline \multirow[t]{2}{*}{ Walking speed } & placebo group $n=6$ & $18.1 p=0.041$ & & & & $37.1 p=0.032$ \\
\hline & active treatment $n=14$ & & 12.2 & 17.3 & 17.7 & $24.8 p<0.001$ \\
\hline \multirow[t]{2}{*}{ Dual support, stance phase of the right leg } & placebo group $n=6$ & $6.1 p=0.093$ & & & & $12.6 p=0.463$ \\
\hline & active treatment $n=14$ & & 4.2 & 7 & 6.8 & $12.2 p=0.009$ \\
\hline
\end{tabular}

however, there was still an alleviation of pain of around $50 \%$ to be recorded. This improvement of quality of life is the most important result for the patients.

The measurement values of the motor parameters reveal a development that is not easy to interpret. During the 2 weeks of treatment with ESW, an improvement takes place that goes beyond the placebo effect. Afterwards there is once again a slight worsening over a two-week period, which then recedes in the face of a renewed improvement over the subsequent 4 weeks.

High- and medium-energy ESW can destroy nonmyelinated nerves, which then regenerate even faster (Ohtori, 2013). However, this requires much greater energies (Hausdorf 1, 2008; Hausdorf 2, 2008; Ohtori, 2013) over topographically more limited areas than those we used for our treatment. We keep the therapy source in constant movement over the entire sole of the foot during the therapy in order to avoid a cumulation of potentially damaging energy. In the rat, 3 weeks are specified for the regeneration of peripheral nerves with low energy EWST (Mense, 2013). No damaging influence on the nociceptive system appears to originate from this energy level (Haake, 2002). The phenomenon of the alleviation of feelings of discomfort and pain from ESW in cases of DSPNP that we observed remains unexplained.

Independently of these considerations, the patients nevertheless still experienced an alleviation of their subjective complaints of more than $50 \%$, which then continued beyond the actual treatment time itself. Those who have fewer complaints during movement will also move more. A training effect on muscle strength and proprioception while walking is thus also to be considered. Muscle build-up leads to acceleration while walking. The improvement of coordination results in improved balance and thus a shortening of the dualsupport time while walking.

While the regeneration of crushed sciatic nerves and electrophysiological parameters (Mense, 2013) in the rat took three weeks with the aid of ESWT, the func- tional gain with respect to the mobility of the animals did not reveal itself until after six weeks had passed. Whether a partial regeneration of DSPNP-damaged nerves is actually accomplished in humans remains to be demonstrated.

The technical equipment of a large neurology department is required for further clarification of the phenomena and for dosage determination.

The investigation was carried out with the limited means of a small department in a rehabilitation clinic. The number of cases was too small to reach binding conclusions. Nonetheless, the effect of ESWT on gait economy and on neuropathic feelings of discomfort can be demonstrated.

\section{Acknowledgments}

The non-profit association "Mobilitas e. V." funded the assessments and the publication fee. Prof. Dr. Toni Graf-Baumann funded the device for measuring the kinematics.

\section{Declaration of interest}

The first author received a research grant from Storz Medical, Switzerland. The second author is an employee of Storz Medical, Switzerland.

\section{References}

Al-Abbad, H., \& Simon, J. V. (2013). The effectiveness of extracorporeal shock wave therapy on chronic achilles tendinopathy: A systematic review. Foot Ankle Int, 34, 33-41.

Amelio, E., \& Manganotti, P. (2010). Effect of shock wave stimulation on hypertonic plantar flexor muscles in patients with cerebral palsy: A placebo-controlled study. J Rehabil Med, 42, 339-343.

Gutersohn, A., \& Gaspari, G. (2000). Shock waves upregulate vascular endothelial growth factor m-RNA in human umbilical vascular endothelial cells. Circulation, 102(Suppl I), 1-18. 
Haake, M., Thon, A., \& Bette, M. (2002). No influence of low-energy extracorporeal shock wave therapy (ESWT) on spinal nociceptive systems. J Orthop Sci, 7, 97-101.

Hausdorf, J., Lemmens, M. A. M., Heck, K. D. W., Grolms, N., Korr, H., Kertschanska, S., Steinbusch, H. W. M., Schmitz, C., \& Maier, M. (2008). Loss of unmyelinated nerve fibers after extracorporeal schockwave application to the musculoskeletal system. Neurosci, 155, 138-144.

Hausdorf, J., Lemmens, M. A. M., Kaplan, S., Marangoz, C., Milz, S., Odaci, E., Korr, H., Schmitz, C., \& Maier, M. (2008). Extracorporeal shockwave application to the distal femur of rabbits diminishes the number of neurons immunoreactive for substance $\mathrm{P}$ in dorsal root ganglia L5. Brain Res, 1207, 96-101.

Hausner, T., Pajer, K., Halat, G., Hopf, R., Schmidhammer, R., Redl, H., \& Nógrádi, A. (2012). Improved rate of peripheral nerve regeneration induced by extracorporeal shock wave treatment in the rat. Exp Neurol, 236, 363-370.

Ito, K., Fukumoto, Y., \& Shimokawa, H. (2009). Extracorporeal shock wave therapy as a new and non-invasive angiogenic strategy. Tohoku J Exp Med, 219, 1-9.

Jin, K., Mao, X. O., \& Greenberg, D. A. (2006). Vascular endothelial growth factor stimulates neurite outgrowth from cerebral cortical neurons via Rho kinase signaling. J Neurobiol, 15, 236-242.

Kato, K., Fujimura, M., Nakagawa, A., Saito, A., Ohki, T., Takayama, K., \& Tominaga, T. (2007). Pressure-dependent effect of shock waves on rat brain: Induction of neuronal apoptosis mediated by a caspase-dependent pathway. J Neurosurg, 106, 667-676.

Kubo, M., Li, T. S., Kamota, T., Ohshima, M., Shirasawa, B., \& Hamano, K. (2010). Extracorporeal shock wave therapy ameliorates secondary lymphedema by promoting lymphangiogenesis. $J$ Vasc Surg, 52, 429-434.

Laird, J. M., Mason, G. S., Thomas, K. A., Hargreaves, R. J., \& Hill, R. G. (1995). Acidic fibroblast growth factor stimulates motor and sensory axon regeneration after sciatic nerve crush in the rat. Neuroscience, 65, 209-216.

Lohse-Busch, H., Kraemer, M., \& Reime, U. (1997). A pilot investigation into the effects of extracorporeal shock waves on muscular dysfunction in children with spastic movement disorders. Schmerz, 18, 108-112.

Ma, H. Z., Zeng, B. F., \& Li, X. L. (2005). Upregulation of VEGF in subchondral bone of necrotic femoral heads in rabbits with use of extracorporeal shock waves. Calcif Tissue Int, 81, 124-131.

Manganotti, P., \& Amelio, E. (2005). Long term effect of shock wave therapy on upper limb hypertonia in patients affected by stroke. Stroke, 36, 1967-1971.

Mariotto, S. W., Cavalieri, E., Amelio, E., Ciampa, A. R., Carcereri de Prati, A., Marlinghaus, E., Russo, S., \& Suzuki, H. (2005). Extracorporeal shock waves: From lithotripsy to anti- inflammatory action by NO production. Nitric Oxide, 12, 89-96.

Mariotto, S., de Prati1, A. C., Cavalieri1, E., Amelio, E., Marlinghaus, E., \& Suzuki, H. (2009). Extracorporeal shock wave therapy in inflammatory diseases: Molecular mechanism that triggers antiinflammatory action. Curr Med Chem, 16, 2366-2372.

Melikyan, E. Y., Shahin, E., Miles, J., \& Bainbridge, L. C. (2003). Extracorporeal shock-wave treatment for tennis elbow. A randomised double-blind study. J Bone Joint Surg Br, 85, 852-855.

Mense, S., \& Hoheisel, U. (2013). Shock wave treatment improves nerve regeneration in the rat. Muscle \& Nerve, 47, 702-710.

Mouzopoulos, G., Stamatakos, M., Mouzopoulos, D., \& Tzurbakis, M. (2007). Extracorporeal shock wavetreatment for shoulder calcific tendonitis: A systematic review. Skeletal Radiol, 36, 803811.

Mudò, G., Bonomo, A., Di Liberto, V., Frinchi, M., Fuxe, K., \& Belluardo, N. (2009). The FGF- 2/FGFRs neurotrophic system promotes neurogenesis in the adult brain. J Neural Transm, 116, 995-1005.

Ohtori, S., Inoue, G., Mannoji, C., Saisu, T., Takahashi, K., Mitsuhashi, S., Wada, Y., Takahashi, K., Yamagata, M., \& Moriya, H. (2013). Shock wave application to rat skin induces degeneration and reinnervation of sensory nerve fibers. Neurosci Lett, 15, 57-60.

Serizawa, F., Ito, K. M., Matsubara, A., Sato, A. H., Shimokawa, B., \& Satomi, S. (2011). Extracorporeal shock wave therapy induces therapeutic lymphangiogenesis in a rat model of secondary lymphoedema. Eur J Vasc Endovasc Surg, 42, 254-260.

Speed, C. A., Nichols, D., Wies, J., Humphreys, H., Richards, C., Burnet, S., \& Hazleman, B. L. (2003). Extracorporeal shock wave therapy for plantar fasciitis. A double blind randomised controlled trial. JOrthop Res, 21, 937-940.

Sun, Y., Jin, K., Childs, J. T., Xie, L., Mao, X. O., \& Greenberg, D. A. (2006). Vascular endothelial growth factor-B (VEGFB) stimulates neurogenesis: Evidence from knockout mice and growth factor administration. Dev Biol, 289, 329-335.

Troncatia, F., Pacia, M., Myftarib, T., \& Lombardia, B. (2013). Extracorporeal shock wave therapy reduces upper limb spasticity and improves motricity in patients with chronic hemiplegia: A case series. NeuroRehabilitation, 33, 399-405. DOI:10.3233/NRE130970

Wang, C. J., Yang, Y. J., \& Huang, C. C. (2011). The effects of shockwave on systemic concentrations of nitric oxide level, angiogenesis and osteogenesis factors in hip necrosis. Rheumatol Int, 31, 871-877.

Widenfalk, J., Lipson, A., Jubran, M., Hofstetter, C., Ebendal, T., Cao, Y., \& Olson, L. (2003). Vascular endothelial growth factor improves functional outcome and decreases secondary degeneration in experimental spinal cord contusion injury. Neuroscience, 120, 951-960.

Wu, Y. H., Liang, H. W., Chen, W. S., Lai, J. S., Luh, J. J., \& Chong, F. C. (2008). Electrophysiological and functional effects of shock waves on the sciatic nerve of rats. Ultrasound Med Biol, 34, 16881696 